\title{
Extending extremal contractions from an ample section
}

\author{
Marco Andreatta and Gianluca Occhetta \\ (Communicated by A. Sommese)
}

\begin{abstract}
Let $\mathscr{E}$ be an ample vector bundle of rank $r$ on a complex projective manifold $X$ such that there exists a section $s \in \Gamma(\mathscr{E})$ whose zero locus, $Z=(s=0)$, is a smooth submanifold of the expected dimension $\operatorname{dim} X-r$. We study the problem of extending birational contractions of $Z$ to the ambient variety proving an extension property for blow-ups and we apply our results to classify $X$ as above when $Z$ is a $\mathbb{P}$-bundle on a surface with nonnegative Kodaira dimension.
\end{abstract}

Key words. Vector bundle, extremal ray, Fano-Mori contraction.

2000 Mathematics Subject Classification. Primary 14E30, 14J40; Secondary 14F05

\section{Introduction}

All through the paper we will work in the following

Setup 1.1. Let $X$ be a smooth complex projective variety of dimension $n$ and $\mathscr{E}$ an ample vector bundle of rank $r$ on $X$ such that there exists a section $s \in \Gamma(\mathscr{E})$ whose zero locus, $Z=(s=0)$, is a smooth submanifold of the expected dimension $\operatorname{dim} Z=$ $\operatorname{dim} X-r=n-r$.

A classical and natural problem is to exploit the geometric properties of $Z$ to get information on the geometry of $X$; for an account of the results in case $r=1$, i.e. when $Z$ is an ample divisor, see [6, Chapter 5]. In [3] we considered the problem from the point of view of Mori theory, posing the following question: assume that $Z$ is not minimal, i.e. $Z$ has at least one extremal ray in the negative part of the Mori cone; does this ray (or the associated extremal contraction) determine a ray (or a contraction) in $X$, and if so, does this new ray determine the structure of $X$ ?

Through the paper we will assume that $Z$ is not minimal, we assume also that $\operatorname{dim} Z \geqslant 2$; if $\operatorname{dim} Z=1$ then $Z \simeq \mathbb{P}^{1}$ and this case is treated in [13], where the problem of special sections of an ample vector bundle was studied first.

We showed in [3] that there is always an extremal face $F_{Z}$ of $N E(Z)$ that determines an extremal face $F_{X}$ of $N E(X)$; now we slightly improve our results, proving that, if $N_{1}(Z) \simeq N_{1}(X)$ (which is always true if $\operatorname{dim} Z \geqslant 3$, see 2.8) then there is always an 
extremal face $F_{Z}$ of $N E(Z)$ which coincides with an extremal face of $N E(X)$ and in this case we say that the face is liftable to $X$; this is the context of Theorem 3.2 and Corollary 3.4.

Note that, a priori, the liftability of a face does not imply the extendability of the associated contraction; namely the contraction associated to $F_{X}$ on $X$ restricted to $Z$ is not necessarily the contraction associated to $F_{Z}$ (see Remark 3.5 ); if this is the case we say that the face is extendable. If $F_{Z}$ corresponds to a fiber type contraction on $Z$, then $F_{Z}$ is extendable and the contraction of the face $F_{X}$ in $X$ is again a fiber type contraction [3, 3.12 and 3.13].

In this paper we study the extendability problem for birational contractions: a general result is given in 3.8. Then we prove an extension property for blow-ups, namely:

Theorem 1.2. Let $R_{Z}$ be an extremal ray on $Z$, whose associated contraction $\varphi: Z \rightarrow Z^{\prime}$ is the blow-up of a smooth subvariety $C$ of codimension $m \geqslant 3$.

If $R_{Z}$ is liftable to $R_{X}$ then it is extendable; moreover, if $\phi: X \rightarrow X^{\prime}$ is the contraction associated to $R_{X}$, then $X^{\prime}$ is smooth, $Z^{\prime}$ is isomorphic to a subvariety of $X^{\prime}$, and $\phi$ is the blow-up of $C$.

A straightforward corollary of this theorem is the following

Corollary 1.3. Assume that $\varphi: Z \rightarrow Z^{\prime}$ is the blow-up of a smooth minimal variety $\left(K_{Z^{\prime}}\right.$ is nef) along a smooth subvariety of codimension $m \geqslant 3$. Then $X$ is the blow-up of a smooth variety $X^{\prime}$ along a smooth subvariety of codimension $m+r$.

Another application of the above theorem concerns the problem to determine whether a blow-up of a projective space $\mathbb{P}^{n-r}$ along a linear subspace can be the zero locus of a section of an ample vector bundle in a projective manifold. We discuss this problem in the second part of Section 4.

We finally apply our results to prove the following

Theorem 1.4. Assume that $Z$ is a $\mathbb{P}^{d}$-bundle on a surface $S$ of nonnegative Kodaira dimension. Then $X$ is a $\mathbb{P}^{r+d}$-bundle on $S$.

In the case $r=1$ this theorem, together with previous existing results, completes the proof of a long lasting general conjecture by A. Sommese for $n=4$, see [6, Conjecture 5.5.1]; the same theorem was proved in a different way (only for the case $r=1$ and $n=4)$ recently in [16].

We would like to thank the referee for remarks and suggestions which improved the exposition of the results in the paper. We are both partially supported by grants of the MURST.

\section{Notations and preliminaries}

We use the standard notation from algebraic geometry, in particular it is compatible with that of [11] and of [12]. This paper is a sequel of [3] to which we refer constantly. 
In the paper $X$ will always stand for a smooth complex projective variety of dimension $n$ and $K_{X}$ will be its canonical divisor; the famous Cone Theorem of Mori says that the closure of the cone of effective 1-cycles into the real vector space of 1-cyles modulo numerical equivalence, $\overline{N E(X)} \subset N_{1}(X)$, is polyhedral in the part contained in the set $\left\{Z \in N_{1}(X): K_{X} . Z<0\right\}$. An extremal face $F$ (or $F_{X}$ ) of $X$ is a face of this polyhedral part; an extremal ray is a face of dimension 1 . To every extremal face is associated a morphism to a normal variety; namely we have the following Base point free theorem of Kawamata and Shokurov.

Theorem 2.1. Let $X$ and $F$ be as above. Then there exists a projective morphism $\varphi: X \rightarrow W$ from $X$ onto a normal variety $W$ which is characterized by the following properties:

i) For an irreducible curve $C$ in $X, \varphi(C)$ is a point if and only if the class of $C$ is in $F$.

ii) $\varphi$ has only connected fibers.

Definition 2.2. The map $\varphi$ of the above theorem is usually called the Fano-Mori contraction (or the extremal contraction) associated to the face $F$. A Cartier divisor $H$ such that $H=\varphi^{*}(A)$ for an ample divisor on $W$ is called a good supporting divisor of the map $\varphi$ (or of the face $F$ ).

The contraction is of fiber type if $\operatorname{dim} W<\operatorname{dim} X$, otherwise it is birational. We usually denote with $E=E(\varphi):=\left\{x \in X: \operatorname{dim}\left(\varphi^{-1} \varphi(x)\right)>0\right\}$ the exceptional locus of $\varphi$; if $\varphi$ is of fiber type then of course $E:=X$.

Remark 2.3. Note that a good supporting divisor for a Fano-Mori contraction is of the form $H=K_{X}+r L$, where $r$ is a positive integer and $L$ is an ample line bundle. In fact if $H$ is a good supporting divisor then $H-K_{X}$ is an ample line bundle by Kleiman's criterion.

On the other hand note also that any nef but not ample line bundle $H$ of the form $H=K_{X}+r L$, with $r$ a positive integer and $L$ an ample line bundle, defines (or is associated to) an extremal face $F:=\{Z \in \overline{N E(X)}: H . Z=0\}$.

Example 2.4. Let $\varphi: X:=\mathrm{B}_{Y}\left(X^{\prime}\right) \rightarrow X^{\prime}$ be the blow-up of a projective manifold $X^{\prime}$ along a submanifold $Y \subset X^{\prime}$ of codimension $m$; let also $E \subset X$ be the exceptional divisor. This is a Fano-Mori contraction and a good supporting divisor for this contraction is $H=K_{X}+(m-1) L$, where $L=-E+\varphi^{*}(A)$ for an ample divisor $A$ on $X^{\prime}$.

Let $W$ be a projective manifold and let $\mathscr{G}$ be a rank $m$ vector bundle on $W$; then $\varphi: X:=\mathbb{P}(\mathscr{G}) \rightarrow W$ is a Fano-Mori contraction, called a $\mathbb{P}$-bundle contraction or a (classical) scroll over $W$; to avoid possible confusion, we will use always the first denomination. If $\xi_{\mathscr{G}}$ denotes the tautological bundle on $X$ then a good supporting divisor for the contraction $\varphi$ is $H=K_{X}+m L$, where $L=\xi_{\mathscr{G}}+\varphi^{*}(A)$ for an ample divisor $A$ on $W$.

More generally a fiber type contraction $\varphi: X \rightarrow W$ of a projective manifold $X$ onto a normal projective variety $W$ supported by a divisor of the type $H=K_{X}+$ $(\operatorname{dim} X-\operatorname{dim} W+1) L$, with $L$ an ample line bundle, is a Fano-Mori contraction 
and it is called an adjunction theoretic scroll. In a neighborhood of a generic fiber an adjunction theoretic scroll is a P-bundle (this is a theorem of Fujita); however there can be special fibers of dimension greater than $(\operatorname{dim} X-\operatorname{dim} W)$.

Another important result of Mori, see [15], is the existence of rational curves in the extremal rays. Namely if $X$ has an extremal ray $R$ then there exists a rational curve $C$ on $X$ such that $R=\mathbb{R}[C]$ and $0<-K_{X} \cdot C \leqslant n+1$. Such a curve $C$ is called an extremal curve.

Definition 2.5. Let $R$ be an extremal ray on $X$. We define the positive integer $l$ as

$$
l=l(R):=\min \left\{-K_{X} \cdot C: C \text { is a rational curve in } R\right\} .
$$

$l$ is called the length of the ray while a rational curve $C$ in the ray $R$ such that $l=-K_{X} . C$ is called a minimal extremal curve.

The importance of this integer comes from the following proposition, proved by Ionescu and Wisniewski.

Proposition 2.6 ([19]). Let $\varphi$ be an extremal contraction associated to the extremal ray $R$; let $S$ be an irreducible component of a non-trivial fiber of $\varphi$. The following formula holds

$$
\operatorname{dim} S+\operatorname{dim} E(\varphi) \geqslant \operatorname{dim} X+l(R)-1 .
$$

The zero locus of a section of an ample vector bundle has a lot of good properties, we will frequently use the following two:

Proposition $2.7([3,2.18])$. Let $X$ and $Z$ be as in 1.1 and let $Y$ be a subvariety of $X$ of dimension $\geqslant r$. Then $\operatorname{dim} Z \cap Y \geqslant \operatorname{dim} Y-r$.

The other is a very strong result, a Weak Lefschetz type theorem for ample vector bundles, proved by Sommese in [17] and subsequently with slightly weaker assumptions in [13].

Theorem 2.8. Let $X, \mathscr{E}$ and $Z$ be as in 1.1 and let $i: Z \hookrightarrow X$ be the embedding. Then

(2.8.1) $\mathrm{H}^{i}(i): \mathrm{H}^{i}(X, \mathbb{Z}) \rightarrow \mathrm{H}^{i}(Z, \mathbb{Z})$ is an isomorphism for $i \leqslant \operatorname{dim} Z-1$.

(2.8.2) $\mathrm{H}^{i}(i)$ is injective and its cokernel is torsion free for $i=\operatorname{dim} Z$.

(2.8.3) $\operatorname{Pic}(i): \operatorname{Pic}(X) \rightarrow \operatorname{Pic}(Z)$ is an isomorphism for $\operatorname{dim} Z \geqslant 3$.

(2.8.4) $\operatorname{Pic}(i)$ is injective and its cokernel is torsion free for $\operatorname{dim} Z=2$.

The following lemma is probably well known but we will provide anyway a proof for the interested reader, since we did not find a good reference for it. 
Lemma 2.9. Let $\varphi: X \rightarrow X^{\prime}$ be the blow-up of a smooth variety along a smooth subvariety $Y \subset X^{\prime}$ with exceptional divisor $E(\varphi)$ and $\mathscr{E}$ a rank $r$ vector bundle on $X$. Assume that $\mathscr{E}_{F} \simeq \oplus^{r} \mathcal{O}_{\mathbb{P}}(1)$ for every fiber of $\varphi$. Then there exists a rank $r$ vector bundle $\mathscr{E}^{\prime}$ on $X^{\prime}$ such that

$$
\mathscr{E} \otimes E(\varphi)=\varphi^{*} \mathscr{E}^{\prime}
$$

Proof. The vector bundle $\tilde{\mathscr{E}}:=\mathscr{E} \otimes E(\varphi)$ is trivial along any fiber of $\varphi$. We have to prove that $f_{*}(\tilde{\mathscr{E}})=: \mathscr{E}^{\prime}$ is a locally free sheaf of rank $r$. This is a local question at any point $y \in Y$, and we can apply the Theorem on Formal Functions, see [10, Theorem III.11.1], which says that

$$
f_{*}(\tilde{\mathscr{E}})_{y}=\lim _{\longleftarrow} \mathrm{H}^{0}\left(F_{n}, \tilde{\mathscr{E}}_{n}\right)
$$

(with $F_{n}=X \times_{Y} \operatorname{Spec}\left(\mathcal{O}_{y} / m_{y}^{n}\right)$ ). Since $\tilde{\mathscr{E}}$ is trivial on $F=\varphi^{-1}(z)$ there are $r$ linearly independent non-zero sections of $\tilde{\mathscr{E}}_{\mid F}=F \times \mathbb{C}^{r}$; the same proof of the Castelnuovo criterion for blow-up, as for instance in [5, Proposition 2.4] or [10, Theorem V.5.7], gives that the sections actually extend to $r$ non-zero and linearly independent sections of the $\lim \mathrm{H}^{0}\left(F_{n}, \tilde{\mathscr{E}}_{n}\right)$ (i.e. they extend in a formal neighbourhood of $F$ ). This gives that $f_{*} \overleftarrow{(\tilde{\mathscr{E}})_{y}}$ is locally free of rank $r$.

In the setup of the previous lemma, it is useful to find conditions which ensure the ampleness of $\mathscr{E}^{\prime}$ :

Lemma 2.10. In the situation of the previous lemma if $\mathscr{E}$ is ample and $Y$ is a point then also $\mathscr{E}^{\prime}$ is ample; the same is true if $\mathscr{E}$ is ample and there exists a surjection $N_{Y}^{*} \rightarrow$ $-L \rightarrow 0$ with $L$ a nef line bundle on $Y$.

Proof. The first part has been proved in [14, Lemma 5.1]. To prove the second part we will apply [7, Lemma 5.7]; let $E=\mathbb{P}\left(N_{Y}^{*}\right)$ be the exceptional divisor of $\varphi$ and consider the following diagram:

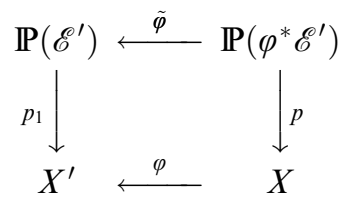

where $\tilde{\varphi}$ is the blow-up of $\mathbb{P}\left(\mathscr{E}^{\prime}\right)$ along $p_{1}^{-1}(Y)$. Let $\xi_{\mathscr{E}^{\prime}}$ be the tautological line bundle of $\mathbb{P}\left(\mathscr{E}^{\prime}\right)$; we have

$$
\tilde{\varphi}^{*} \xi_{\mathscr{E}^{\prime}}=\xi_{\varphi^{*} \mathscr{E}^{\prime}}=\xi_{\mathscr{E} \otimes \mathcal{O}(E)}=\xi_{\mathscr{E}}+p^{*} E
$$

so that $\tilde{\varphi}^{*} \xi_{\mathscr{E}^{\prime}}-p^{*} E=\xi_{\mathscr{E}}$ is ample on $\mathbb{P}\left(\varphi^{*} \mathscr{E}^{\prime}\right)=\mathbb{P}(\mathscr{E})$.

Moreover $\xi_{\mathscr{E}}$ is ample on $p_{1}^{-1}(Y)$. In fact, let $Y^{\prime} \subset E$ be the section correspond- 
ing to the surjection $N_{Y}^{*} \rightarrow-L \rightarrow 0 ; Y^{\prime}$ is mapped isomorphically onto $Y$ by $\varphi$ and $E_{Y^{\prime}}=L_{Y^{\prime}}$, hence we have

$$
(\mathscr{E} \otimes L)_{\mid Y^{\prime}}=(\mathscr{E} \otimes E)_{\mid Y^{\prime}}=\varphi^{*} \mathscr{E}_{\mid Y^{\prime}}^{\prime}=\mathscr{E}_{\mid Y}^{\prime}
$$

and $\mathscr{E}^{\prime}$ is ample on $Y$. We can thus apply the quoted lemma to get the ampleness of $\xi_{\mathscr{E}^{\prime}}$.

\section{Lifting of birational contractions}

Let $X, \mathscr{E}$ and $Z$ be as in 1.1; in this section we will improve some general results in [3]; we start with a definition which was not stated there.

Definition 3.1. Assume that $N_{1}(Z) \simeq N_{1}(X)$, which is always the case if $\operatorname{dim} Z \geqslant 3$ by Theorem 2.8, and let $F_{Z}$ be an extremal face in $N E(Z)$. If under the above identification $N_{1}(Z) \simeq N_{1}(X)$ the face $F_{Z}$ is an extremal face $F_{X}$ in $N E(X)$, then we will say that the face $F_{Z}$ is liftable to $F_{X}$.

The following is a refinement of [3, Theorem 3.4]:

Theorem 3.2 (Lifting of extremal faces). Assume that $Z$ is not minimal in the sense of Mori theory, i.e. $K_{Z}$ is not nef. Let $F_{Z}$ be an extremal face of $Z, D_{Z}=\left(K_{Z}+\tau H_{Z}\right)$ a good supporting $\mathbb{Q}$-Cartier divisor of $F_{Z}$ and $H$ the line bundle on $X$ which restricts to $H_{Z}$. If $H$ is ample on $X$, the $\mathbb{Q}$-divisor $D=K_{X}+\operatorname{det} \mathscr{E}+\tau H$ is nef, not ample and defines an extremal face $F_{X}$ of $X$.

Moreover, if $N_{1}(Z) \simeq N_{1}(X), F_{Z}$ is liftable to $F_{X}$.

Proof. The first part of the theorem has been proved in [3, Theorem 3.4], so we have to prove only the last assertion. Since under the identification $N_{1}(Z) \simeq N_{1}(X)$ we have $N E(Z) \subset N E(X)$ and $N E(Z)_{K<0} \subset N E(X)_{K<0}$ it is enough to show that, for every extremal ray $R_{X}$ in the face $F_{X}$, there is a curve in $R_{X}$ lying on $Z$.

Let $R_{X}$ be an extremal ray of $F_{X}$ and $\varphi_{R}: X \rightarrow T$ the associated extremal contraction; since the contraction of $F_{X}$ is supported by $K_{X}+\operatorname{det} \mathscr{E}+\tau H$, this divisor is zero on the curves in $R_{X}$, yielding $l\left(R_{X}\right) \geqslant r+\tau$; if the contraction is birational, then, using 2.6, for a non-trivial fiber $F$ of $\varphi_{R}, \operatorname{dim} F \geqslant r+\tau$, hence $\operatorname{dim} F \geqslant r+1$ and we are done by 2.7 .

In the same way we get our result if the contraction is of fiber type and has a fiber of dimension $r+1$; so we are left with the case of an equidimensional fiber type contraction with $r$-dimensional fibers; note that in this case, the $\varphi_{R}$-ample line bundle $H$ has intersection number one with the extremal rational curve generating the ray by [3, Proposition 2.7], so that, letting $H^{\prime}=H+\varphi_{R}^{*} A$, with $A$ ample on $T$, the divisor $K_{X}+(r+1) H^{\prime}$ is a good supporting divisor for $\varphi_{R}$, which, by $[8,2.12]$ is thus a $\mathbb{P}$ bundle contraction.

As in the proof of the first part of [3, Theorem 3.4], we get that $Z$ is a regular section of this bundle, a contradiction with Theorem 2.8 . 
Proposition 3.3. Assume that $N_{1}(Z) \simeq N_{1}(X)$ and that the extremal face $F_{Z}$ is liftable to $F_{X}$. Denote by $\varphi$ and $\phi$ the contractions associated to $F_{Z}$ and $F_{X}$; let $K_{Z}+\tau H_{Z}$ be a good supporting divisor for $F_{Z}$ and let $H$ be the divisor on $X$ which restricts to $H_{Z}$.

Then, up to replacing $H$ with $H^{\prime}=H+\phi^{*} A$, with $A$ a sufficiently ample line bundle, we can assume that that $H^{\prime}$ is ample on $X$ and that $\varphi$ is supported by $K_{Z}+\tau H_{Z}^{\prime}$.

Proof. The line bundle $H$ is $\phi$-ample and thus $H^{\prime}$ is ample. Moreover $K_{Z}+\tau H_{Z}^{\prime}=$ $K_{Z}+\tau H_{Z}+\tau\left(\phi^{*} A\right)_{Z}$ is a good supporting divisor of $F_{Z}$ since $\tau\left(\phi^{*} A\right)_{Z}$ is nef and it is zero on the curves of $F_{Z}$.

Proposition 3.4. If $Z$ is not minimal there exists at least one extremal face $F_{Z}$ which is liftable to $X$.

Proof. Let $L$ be an ample line bundle on $X$; the restriction of this line bundle to $Z$, $L_{Z}$, is ample on $Z$, so, if $K_{Z}$ is not nef there exist a rational number $\sigma>0$ such that the divisor $K_{Z}+\sigma L_{Z}$ is nef but not ample and it defines an extremal face $G_{Z}$. This face satisfies the assumptions of Theorem 3.2 and so it is liftable to an extremal face $G_{X}$.

Remark 3.5. Let us note that, a priori, the fact that an extremal face of $\overline{N E(Z)}$ is liftable to an extremal face of $\overline{N E(X)}$ does not imply that the restriction $\phi_{Z}$ of the extremal contraction $\phi$ associated to $F_{X}$ coincides with the extremal contraction $\varphi$ associated to $F_{Z}$; as explained in [3], we have a commutative diagram

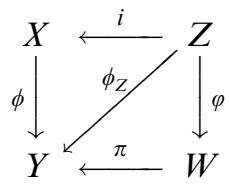

where $\pi: W \rightarrow \phi_{Z}(Z)$ is a finite morphism.

To complete the lifting process, we introduce the following definition:

Definition 3.7. In the above notation, if $\pi$ is a isomorphism onto its image, that is if the restriction $\phi_{Z}$ coincides with the extremal contraction $\varphi$ of $F_{Z}$, then we will say that the face $F_{Z}$, or the associated contraction $\varphi$, is extendable.

In [3] we proved that if $F_{Z}$ is a liftable face associated to a fiber type contraction then it is extendable and moreover $\pi$ is the identity. Now we will deal with birational contractions.

Proposition 3.8. Assume that there exists an extremal ray $R_{Z}$, whose associated contraction, $\varphi: Z \rightarrow W$, is birational, which is liftable to an extremal ray $R_{X}$. We can assume that $\varphi$ is supported by $K_{Z}+\tau H_{Z}$ with $\tau \geqslant 1$ (Remark 2.3) and that $H_{Z}$ is the restriction of an ample line bundle $H$ on $X$ (Proposition 3.3). 
If $\tau>1$ then $\phi$ is birational, $\phi_{Z}$ has connected fibers and $\pi: W \rightarrow \phi_{Z}(Z)$ is the normalization morphism. If $\tau=1$ then $\phi$ can be either birational or of fiber type; in the first case $\phi_{Z}$ has connected fibers and $\pi: W \rightarrow \phi_{Z}(Z)$ is the normalization morphism while in the second $\phi$ is an adjunction theoretic scroll contraction onto $W$ (see 2.4) and $R_{Z}$ is extendable.

Proof. If $\tau>1$ the proof is as in [3, Propositions 3.13, 3.14], observing that the intersection of $Z$ with any non-trivial fiber $F$ of $\phi$ has $\operatorname{dimension} \operatorname{dim}(Z \cap F) \geqslant 1$.

If $\tau=1$, by Theorem 3.2 the contraction $\phi$ is supported by $K_{X}+\operatorname{det} \mathscr{E}+H$, and so its length $l(\phi)$ is $\geqslant r+1$.

If $\phi$ is birational then again the proof of [3, Propositions 3.13] applies since $\operatorname{dim}(Z \cap F) \geqslant 1$.

If $\phi$ is of fiber type, by Inequality 2.6 we have that all its fibers have dimension $\geqslant r$; if the generic fiber has dimension $\geqslant r+1$ then it has non-trivial intersection with $Z$, and this is impossible as in the proof of [3, Proposition 3.14]. So the generic fiber of $\phi$ is $r$-dimensional, $l(\phi)=r+1$ and, if $C$ is a minimal extremal rational curve in a fiber of $\phi$ with $-K_{X} . C=l(\phi)$, then $H . C=1$ and $\operatorname{det} \mathscr{E} . C=r$. In particular $K_{X}+(r+1) H$ is a good supporting divisor for $\phi$, which thus is an adjunction theoretic scroll.

We have to prove now that $R_{Z}$ is extendable; for this we will first prove that $\phi_{Z}$ has connected fibers and then that $\phi_{Z}(Z)$ is normal. Since $\phi_{Z}$ contracts only the curves whose numerical class is in $R_{Z}$, outside of the exceptional locus $E(\varphi) \phi_{Z}$ is finite-toone; in particular, if $f$ is a fiber of $\phi$ which does not contain curves of $E(\varphi)$ then $f$ is $r$-dimensional, and thus is a projective space $\mathbb{P}^{r}$.

Since det $\mathscr{E} . C=r$ for a minimal extremal rational curve, for every line in $f$, $(\operatorname{det} \mathscr{E})_{l} \simeq \mathcal{O}_{\mathbb{P}^{1}}(r), \mathscr{E}_{f}=\oplus^{r} \mathcal{O}(1)$, and $Z \cap f$ is one point, thus it is connected (note that we have proved that, outside of $\phi^{-1}(\phi(E(\varphi))) \phi$ is a projective bundle and $Z$ is a regular section).

On the other hand, the non-trivial fibers of $\phi_{Z}$ are connected since they are intersections of $Z$ with fibers of $\phi$ and $[3,3.13]$ applies again. Thus $\phi_{Z}$ has connected fibers and $\phi_{Z}(Z)=\phi(X)$, which is normal; so $\pi: W \rightarrow \phi_{Z}(Z)$ is the identity and $R_{Z}$ is extendable.

Example 3.9. Let us note that the last case of the above proposition is effective: let $X=\mathbb{P}^{2} \times \mathbb{P}^{1}$ and $Z$ be a $\mathbb{F}_{1}$-surface in the linear system $\mathcal{O}_{\mathbb{P} \times \mathbb{P}}(1,1)$; the contraction of the $(-1)$ curve of $Z$ lifts to the $\mathbb{P}$-bundle contraction onto $\mathbb{P}^{2}$.

Proposition 3.10. In the setup of the above proposition if $\varphi$ and $\phi$ are both birational, then $E(\varphi)=E(\phi) \cap Z$.

Proof. If $x \in E(\varphi)$, then there exists a curve $C \subset Z$ which contains $x$ and is contracted by $\varphi$; but, on $Z, \varphi$ and $\phi_{Z}$ contract the same curves, therefore $x \in C$ is contained in $E(\phi)$.

On the other hand, if $x \in E(\phi) \cap Z$ we consider the unsplit family $V$ of deformations of a minimal extremal rational curve contracted by $\phi$ (see $[12$, IV.2]). If $\operatorname{Locus}(V, 0 \rightarrow x)$ denotes the locus of the curves in $V$ which pass through $x$, by [12, 
IV.2.6], $\operatorname{dim} \operatorname{Locus}(V, 0 \rightarrow x) \geqslant r+\tau$, hence $\operatorname{dim}(\operatorname{Locus}(V, 0 \rightarrow x) \cap Z) \geqslant 1$, so that $x$ lies in a curve contracted by $\phi$, and so by $\varphi$.

Remark 3.11. Actually, the proof of the last proposition shows that the fibers of $\varphi$ are exactly the intersections of the fibers of $\phi$ with $Z$.

\section{Blow-ups}

Proof of Theorem 1.2. Let $D_{Z}=K_{Z}+(m-1) H_{Z}$ be a good supporting divisor of $\varphi: Z \rightarrow Z^{\prime}$, where $H_{Z}$ is an ample line bundle on $Z$ which restricts to $\mathcal{O}_{\mathbb{P}}(1)$ on every non-trivial fiber of $\varphi$. By the Proposition 3.3 we can assume that the extension of $H_{Z}$ to $X$, namely $H$, is ample.

Let as usual $\phi$ be the contraction associated to the ray $R_{X}$ to which $R_{Z}$ is liftable; it is supported by $D=K_{X}+\operatorname{det} \mathscr{E}+(m-1) H$ and, by 3.8 , it is birational.

The non-trivial fibers of $\phi$ have dimension $\leqslant r+m-1$ by Proposition 2.7 (see also Remark 3.11); on the other hand, by Proposition 2.6 the dimension of any non-trivial fiber is exactly $r+m-1$ and $l(\phi)=r+m-1$.

We can apply [2, Theorem 5.2] to deduce that $\phi: X \rightarrow X^{\prime}$ is the blow-up of a smooth subvariety of codimension $r+m-1$. Let us point out also that the restriction of det $\mathscr{E}$ to every line in a fiber of $\phi$ is $\mathcal{O}_{\mathbb{P}^{1}}(r)$, and so $\mathscr{E}$ splits on the fibers of $\phi$ as $\oplus^{r} \mathcal{O}_{\mathbb{P}}(1)$; thus by Lemma 2.9 , we have that $\mathscr{E} \otimes[-E(\phi)]=\phi^{*} \mathscr{E} \prime$.

We want to prove now that $Z^{\prime} \rightarrow X^{\prime}$ is a closed embedding, that is that $R_{Z}$ is extendable. For this, in the spirit described in the introduction of the paper [4] we now consider a local situation: choose a point $z \in \phi_{Z}(E(\varphi))$, an affine neighbourhood $U$ of $z$ in $X^{\prime}$ and consider the restrictions of $\phi$ and $\varphi$ to the inverse images of $U$; to simplify the notation denote again by $X, X^{\prime}$ and $Z$ the new spaces and by $\phi, \phi_{Z}$ and $\varphi$ the restricted maps.

In this affine situation and in the notation of the Lemma 2.9 we have that $\mathscr{E}^{\prime}$ is trivial and in particular $\mathscr{E}$ splits as $\oplus^{r} L$, where $L=-E(\phi)$; note that $K_{X}+(r+m-1) L$ is a good supporting divisor for $\phi$. We will now use the horizontal slicing procedure ([4, Lemma 2.6]): let $L_{i}$, with $i=1, \ldots, r$, be general smooth sections of $L$ and let $X_{i}=$ $\bigcap_{j=1, \ldots, i} L_{j}$; note that $X_{0}=X$ and that $X_{r} \simeq Z$; we have a chain of surjections

$$
\mathrm{H}^{0}(X, D) \rightarrow \mathrm{H}^{0}\left(X_{1}, D_{X_{1}}\right) \rightarrow \cdots \rightarrow \mathrm{H}^{0}\left(Z, D_{Z}\right)
$$

and this implies (see the proof of [4, Lemma 2.6]) that $\pi: Z^{\prime} \rightarrow X^{\prime}$ is a closed embedding.

As mentioned in the introduction we have also the following application.

Proposition 4.1. Assume that $Z$ is the blow-up of a projective space $\mathbb{P}^{n-r}$ along a linear space $Y$ of codimension $m \geqslant 3$. Then $X$ is a projective bundle on $\mathbb{P}^{m-1}$; namely $g: X=$ $\mathbb{P}(\mathscr{G}) \rightarrow \mathbb{P}^{m-1}$ for some vector bundle $\mathscr{G}$ on $\mathbb{P}^{m-1}$ with

$$
0 \rightarrow \mathscr{V} \rightarrow \mathscr{G} \rightarrow \oplus^{n-r-m-1} \mathcal{O}_{\mathbb{P}} \oplus \mathcal{O}_{\mathbb{P}}(1) \rightarrow 0
$$

and $\mathscr{E}=\xi_{\mathscr{G}} \otimes g^{*} \mathscr{V}^{\sim}$, where $\xi_{\mathscr{G}}$ is the tautological line bundle of $\mathscr{G}$. 
Proof. The variety $Z$ has two extremal rays: the blow-down contraction to $\mathbb{P}^{n-r}$ and a $\mathbb{P}$-bundle contraction on $\mathbb{P}^{m-1}$; by Proposition 3.4 one of these rays is liftable to $X$; if the fiber type ray is liftable, then we are done by [3, Corollary 4.2]; as stated in that corollary the existence of the sequence is a known fact about vector bundles (see for instance $[9$, B.5.6]).

We will now show that the birational ray cannot be liftable. Suppose, by contradiction, that this is the case: by Theorem 1.2 we have that the associated contraction is extendable to a contraction $\phi: X \rightarrow X^{\prime}$ which gives a commutative diagram

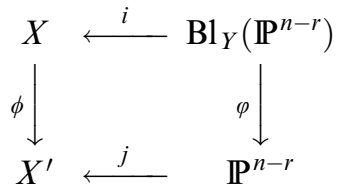

We also know that $\mathscr{E}_{E}=\oplus^{r} \mathcal{O}(1)$; hence there exists a vector bundle $\mathscr{E}^{\prime}$ on $X^{\prime}$ such that $\mathscr{E}=\phi^{*} \mathscr{E}^{\prime} \otimes(-E)$; this vector bundle is ample, by Lemma 2.10 , and $\mathbb{P}^{n-r}$ is the zero locus of a section of it. This implies, by [13, Theorem $\mathrm{A}]$, that $X^{\prime}$ is a projective space $\mathbb{P}^{n}$ and $\mathscr{E}^{\prime}$ decomposes as $\oplus^{r} \mathcal{O}_{\mathbb{P}^{n}}(1)$, but this contradicts the ampleness of $\mathscr{E}$.

Remark 4.2. Let us note that a blow-up of a projective space $\mathbb{P}^{n-r}$ along a linear space $Y$ of codimension $m \geqslant 3$ cannot be an ample section of a line bundle or of a vector bundle which is a direct sum of line bundles; this follows from [7, Proposition 5.8]. Therefore there exists no example for the above proposition if $\mathscr{E}$ is a line bundle (that is if $\mathscr{V}$ is a line bundle), or a direct sum of line bundles.

For general vector bundles $\mathscr{E}$ however this can happen, as the following example will show.

Example 4.3. On $X=\mathbb{P}^{k} \times \mathbb{P}^{2}=\mathbb{P}\left(\oplus^{k+1} \mathcal{O}_{\mathbb{P}^{2}}\right)$, with $k \geqslant 3$, consider the line bundles $\pi_{1}^{*}(\mathcal{O}(a)) \otimes \pi_{2}^{*}(\mathcal{O}(b))=:(a, b)$, where $\pi_{i}$ are the projections. For $m \gg 0$, let $D_{1} \in|(1, m)|$ and $D_{2} \in|(1, m+1)|$ be sufficiently general divisors. They correspond to sections of $\oplus^{k+1} \mathcal{O}_{\mathbb{P}^{2}}(m)$ and $\oplus^{k+1} \mathcal{O}_{\mathbb{P}^{2}}(m+1)$ on $\mathbb{P}^{2}$ and therefore they will give an injective morphism of vector bundles, with cokernel $V$ :

$$
0 \rightarrow \mathcal{O}_{\mathbb{P}^{2}}(-m-1) \oplus \mathcal{O}_{\mathbb{P}^{2}}(-m) \rightarrow \oplus^{k+1} \mathcal{O}_{\mathbb{P}^{2}} \rightarrow V \rightarrow 0 .
$$

We notice that $V$ is actually a vector bundle since, if $k \geqslant 3$, the two sections can be taken linearly independent at each point of $\mathbb{P}^{2}$.

Moreover we have that $V$ is ample; in fact the tautological bundle of $\mathbb{P}(V)$ is the restriction of $\xi=\pi_{1}^{*}(\mathcal{O}(1))$, the tautological bundle of $X$, to $\mathbb{P}(V)$ and therefore our claim follows if we show that the restriction of $\pi_{1}$ to $\mathbb{P}(V)$ is a finite-to-one map onto $\mathbb{P}^{k}$, by a general choice of the sections. Since $\mathbb{P}(V)=D_{1} \cap D_{2}$, this can be proved applying twice the next lemma; the first time to $\pi_{1}: X \rightarrow \mathbb{P}^{k}$ and $L=|(1, m)|$, the second time to $\pi_{1}: D_{1} \rightarrow \mathbb{P}^{k}$ and $L=|(1, m+1)|_{D_{1}}$. Note in fact that, for $m \gg 0$, $\pi_{1 *}((1, m))=S^{m}\left(\oplus^{3} \mathcal{O}_{\mathbb{P}^{k}}\right)(1)$ is a spanned vector bundle on $\mathbb{P}^{k}$ of rank $>k$. 
Lemma 4.4. Let $p: X \rightarrow Y$ be a flat morphism of projective manifolds and let $L$ be an ample and spanned line bundle on $X$. Suppose moreover that $p_{*} L$ is spanned by global sections on $Y$ and that $\operatorname{rank}\left(p_{*} L\right)$ is bigger than $\operatorname{dim} Y$. Then the restriction of $p$ to $a$ general $D \in|L|$ is equidimensional, hence flat.

Proof. It is enough to show that $D$ meets any fiber of the map $p$ properly. In fact $D$ is ample and therefore it meets any fiber; if a fiber $p^{-1}(x)$ is contained in $D$ then it means that the section corresponding to $D$ in $p_{*} L$ will vanish at the point $x$, but this is impossible since the assumptions imply that a general section of $p_{*} L$ does not vanish anywhere.

Now, dualizing the sequence we have constructed on $\mathbb{P}^{2}$ and twisting it by $\mathcal{O}(-m)$ we get

$$
0 \rightarrow V^{\curlyvee}(-m) \rightarrow \oplus^{k+1} \mathcal{O}_{\mathbb{P}^{2}}(-m) \rightarrow \mathcal{O}_{\mathbb{P}^{2}}(1) \oplus \mathcal{O}_{\mathbb{P}^{2}} \rightarrow 0 .
$$

If we set $\mathscr{V}:=V^{\curlyvee}(-m)$ and $\mathscr{G}:=\oplus^{k+1} \mathcal{O}_{\mathbb{P}^{2}}(-m)$ then this is a sequence as in Proposition 4.1: in fact $\xi_{\mathscr{G}} \otimes \pi_{2}^{*} \mathscr{V}^{\sim}=\pi_{1}^{*} \mathcal{O}(1) \otimes \pi_{2}^{*} V$ is an ample vector bundle.

Let therefore $X=\mathbb{P}(\mathscr{G})=\mathbb{P}\left(\oplus^{k+1} \mathcal{O}_{\mathbb{P}^{2}}(-m)\right), \quad g\left(=\pi_{2}\right): X \rightarrow \mathbb{P}^{2}$ and $\mathscr{E}=$ $\xi_{\mathscr{G}} \otimes g^{*} \mathscr{V}^{*}$. Then $\mathscr{E}$ is an ample vector bundle on $X$ with a section $s$, which corresponds to the composite of the duals of the canonical map $g^{*}(\mathscr{G}) \rightarrow \xi_{\mathscr{G}}$ and of $g^{*} \mathscr{V} \rightarrow g^{*} \mathscr{G}$, whose zero locus is $Z:=\mathbb{P}\left(\mathcal{O}_{\mathbb{P}^{2}}(1) \oplus \mathcal{O}_{\mathbb{P}^{2}}\right)$, which is the blow-up of $\mathbb{P}^{3}$ at a point.

\section{$5 \quad \mathbb{P}^{d}$-bundles on surfaces with $\kappa>0$ and their Mori cone}

Proposition 5.1. Let $p: Z \rightarrow S$ be a $\mathbb{P}^{d}$-bundle over a smooth surface such that $\kappa(S) \geqslant 0$; assume that $Z$ has an extremal ray $R$ different from the one associated to the $\mathbb{P}$-bundle contraction. Then the associated contraction $\varphi_{R}$ is a blow-down $\varphi_{R}: Z \rightarrow Z_{1}$ of a divisor $E=p^{-1}(C)$, with $C$ an exceptional $(-1)$-curve on $S$, such that $E \simeq \mathbb{P}^{1} \times \mathbb{P}^{d}$ and $E_{E} \simeq \mathcal{O}(0,-1)$.

Moreover, $Z_{1}$ has a $\mathbb{P}^{d}$-bundle structure on $S_{1}$, where $S_{1}$ is the surface obtained contracting the exceptional curve $C$ on $S$, and $\varphi_{R}(E)$ is a fiber of $p_{1}: Z_{1} \rightarrow S_{1}$.

Proof. Suppose that $Z$ has an extremal ray, $R$, different from the bundle contraction; there exists a rational curve $C_{0}\left(\left[C_{0}\right] \in R\right)$ such that $-K_{Z} \cdot C_{0}>0$ and $p\left(C_{0}\right)$ is not a point. Let $C=p\left(C_{0}\right)$, let $v: \mathbb{P}^{1} \rightarrow C$ be the normalization of $C$ and consider the fiber product

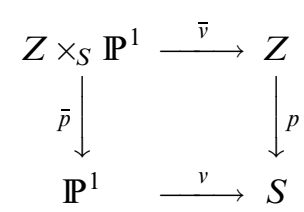

The map $\bar{p}: Z_{C}:=Z \times_{S} \mathbb{P}^{1} \rightarrow \mathbb{P}^{1}$ is a $\mathbb{P}$-bundle on $\mathbb{P}^{1}$; let $C_{0}$ be a minimal section of $\bar{p}$; the proof of [18, Lemma 1.5] applies and we get $K_{S} . C<0$. 
Since on $S$ there is only a finite number of curves which have negative intersection with $K_{S}$ (the $(-1)$-curves) we deduce that the image of the exceptional locus of $\varphi_{R}$ is $C$, and $C$ is a $(-1)$-curve.

Moreover, since the fibers of different extremal contractions can meet only in points, we have that all the fibers of $\varphi_{R}$ have dimension one; combining these facts we get that $\varphi_{R}$ is a divisorial contraction. By [1] $\varphi_{R}$ is a smooth blow-down contraction.

The exceptional locus of $\varphi_{R}, E$, is thus $p^{-1}(C)$ and carries two different $\mathbb{P}$-bundle structures, it is so forced to be $\mathbb{P}^{1} \times \mathbb{P}^{d}$; the description of $E_{E}$ is clear observing that the lines in one ruling are extremal curves for the blow-up, while those in the other ruling are contained in fibers of the bundle projection.

Let $\mathscr{F}$ be a rank $d+1$ vector bundle on $S$ such that $Z=\mathbb{P}_{S}(\mathscr{F})$; the restriction of $\mathscr{F}$ to $C$ is, up to twist, $\oplus^{d+1} \mathcal{O}_{\mathbb{P}^{1}}$; therefore if we denote by $\sigma: S \rightarrow S_{1}$ the contraction of $C$, by Lemma 2.9 there exists a rank $d+1$ vector bundle $\mathscr{F}_{1}$ on $S_{1}$ such that $\mathscr{F}=\sigma^{*} \mathscr{F}_{1}$. Consider the commutative diagram

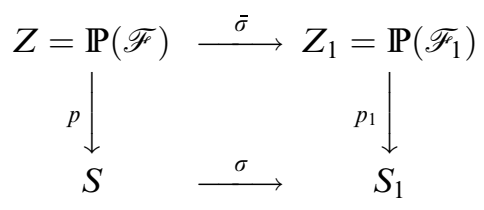

The map $\bar{\sigma}$ is a good contraction which contracts exactly the curves in $R$, so it coincides with $\varphi_{R}$.

\section{$6 \quad \mathbb{P}^{d}$-bundles on surfaces as ample sections}

Proof of Theorem 1.4. By Proposition 5.1 the extremal rays of $Z$ are the ray corresponding to the $\mathbb{P}^{d}$-bundle fibration and, possibly, other rays of birational type; such a ray corresponds to a blow-down $\beta: Z \rightarrow Z_{1}$ which contracts $\mathbb{P}^{d} \times \mathbb{P}^{1}$ to $Y \simeq \mathbb{P}^{d}$.

By Proposition 3.4 we have that at least one extremal ray of $Z$ is liftable to $X$; if this ray is the fiber type one, then, by [3, Corollary 4.2] $X$ is a $\mathbb{P}^{r+d}$-bundle on $S$ and we are done.

Suppose now that the ray that is liftable is a birational one, corresponding to a blowdown $\beta: Z \rightarrow Z_{1}$; by Proposition $5.1 Z_{1}$ has a $\mathbb{P}^{d}$-bundle structure over a smooth surface $S_{1}, p_{1}: Z_{1} \rightarrow S_{1}$, obtained contracting a $(-1)$-curve of $S$ to a point $s_{1}$.

Now $\beta$ is supported by $K_{Z}+H_{Z}$ (e.g. taking $H_{Z}=-E(\beta)$, where $E(\beta)$ is the exceptional divisor of $\beta$ ) and, by Proposition 3.3 we can assume that the line bundle $H$ which restricts to $H_{Z}$ is ample on $X$. By Proposition 3.8 if $\beta$ is liftable to a fiber type ray, $\phi$, then $\phi$ is a scroll contraction $\phi: X \rightarrow Z_{1}$; the proof of Proposition 3.8 also shows that, outside of $\phi^{-1}(\phi(E(\beta))) \phi$ is a projective bundle and $Z$ is a regular section.

Choose a smooth non-rational curve $B$ in $S_{1}$ which does not contain $s_{1} ; T=p_{1}^{-1}(B)$ is a $\mathbb{P}$-bundle on $B$, and it is not contained in $\phi(E(\beta))$. Denote by $U$ the inverse image of $T$ via $\phi, U=\phi^{-1}(T) ; U$ is a $\mathbb{P}$-bundle on $T$ and $Z \cap U$ is a regular section. Therefore $Z \cap U$ is isomorphic to $T$ and thus $\rho(Z \cap U)=2$; on the other hand $Z \cap U$ is the zero 
locus of a section of the ample vector bundle $\mathscr{E}_{U}$, thus, by Theorem $2.8 \rho(Z \cap U) \geqslant 3$, a contradiction.

So $\beta$ is liftable to a birational ray, corresponding to a contraction $\bar{\beta}: X \rightarrow X_{1}$; by the Theorem (1.2) $\beta$ is extendable and $\bar{\beta}$ is a smooth blow-up of $Y_{1} \subset X_{1}$, such that the restriction of $E(\bar{\beta})$ to $Z$ is $E(\beta)$; thus by Lemma 2.9 there exists a vector bundle $\mathscr{E}_{1}$ on $X_{1}$ such that

$$
\mathscr{E} \otimes \mathcal{O}_{X}(E)=\Pi^{*} \mathscr{E}_{1}
$$

and moreover, by Lemma $2.10, \mathscr{E}_{1}$ is ample.

Summing up, we have replaced the starting triple $(X, \mathscr{E}, Z)$ with a new triple $\left(X_{1}, \mathscr{E}_{1}, Z_{1}\right)$ satisfying the assumptions of the theorem and such that $\rho\left(Z_{1}\right)=\rho(Z)-1$. Thus we can repeat the above procedure, i.e. one of the extremal contractions of $Z_{1}$ is liftable. Since $\rho(Z)$ is finite, at some point of this process we must find some triple $\left(X_{k}, \mathscr{E}_{k}, Z_{k}\right)$ such that the $\mathbb{P}^{d}$-bundle contraction of $Z_{k}$ is extendable to a $\mathbb{P}^{r+d}$-bundle contraction of $X_{k}$ and $\mathscr{E}_{k \mid F} \simeq \oplus^{r} \mathcal{O}(1)$ for every fiber of the bundle contraction.

Let $\bar{\beta}_{k}: X_{k-1} \rightarrow X_{k}$ be the last blow-down contraction and let $F$ be the fiber of the $\mathbb{P}^{r+d}$-bundle contraction of $X_{k}$ which contains $Y_{k}$, the center of $\bar{\beta}_{k}$ (which is $F \cap Z_{k} \simeq \mathbb{P}^{d}$ ). Let $l^{\prime}$ be a line in $F$ which meets $Y_{k}$ transversally and let $l$ be its strict transform in $X_{k-1}$. We have

$$
\left(\mathscr{E}_{k-1}\right)_{l} \simeq\left(\bar{\beta}_{k}^{*} \mathscr{E}_{k}\right)_{l} \otimes \mathcal{O}\left(-E_{k}\right)_{l} \simeq\left(\oplus^{r} \mathcal{O}_{\mathbb{P}^{1}}(1)\right) \otimes \mathcal{O}_{\mathbb{P}^{1}}(-1) \simeq \oplus^{r} \mathcal{O}_{\mathbb{P}^{1}}
$$

contradicting the ampleness of $\mathscr{E}_{k-1}$.

\section{References}

[1] T. Ando, On extremal rays of the higher-dimensional varieties. Invent. Math. 81 (1985), 347-357. MR 87g:14045 Zbl 0554.14001

[2] M. Andreatta, G. Occhetta, Special rays in the Mori cone of a projective variety. Preprint AG/0010101.

[3] M. Andreatta, G. Occhetta, Ample vector bundles with sections vanishing on special varieties. Internat. J. Math. 10 (1999), 677-696. MR 2000g:14024 Zbl 01629311

[4] M. Andreatta, J. A. Wiśniewski, A note on nonvanishing and applications. Duke Math. J. 72 (1993), 739-755. MR 95c:14007 Zbl 0853.14003

[5] M. Andreatta, J. A. Wiśniewski, A view on contractions of higher-dimensional varieties. In: Algebraic geometry - Santa Cruz 1995, 153-183, Amer. Math. Soc., Providence, RI 1997. MR 99f:14020 Zbl 0948.14014

[6] M. C. Beltrametti, A. J. Sommese, The adjunction theory of complex projective varieties. Walter de Gruyter \& Co., Berlin 1995. MR 96f:14004 Zbl 0845.14003

[7] T. Fujita, On the hyperplane section principle of Lefschetz. J. Math. Soc. Japan 32 (1980), 153-169. MR 81c:14005 Zbl 0422.14004

[8] T. Fujita, On polarized manifolds whose adjoint bundles are not semipositive. In: Algebraic geometry, Sendai, 1985, 167-178, North-Holland 1987. MR 89d:14006 Zbl 0659.14002

[9] W. Fulton, Intersection theory. Springer 1984. MR 85k:14004 Zbl 0541.14005

[10] R. Hartshorne, Algebraic geometry. Springer 1977. MR 57 \#3116 Zbl 0367.14001 
[11] Y. Kawamata, K. Matsuda, K. Matsuki, Introduction to the minimal model problem. In: Algebraic geometry, Sendai, 1985, 283-360, North-Holland 1987. MR 89e:14015 Zbl 0672.14006

[12] J. Kollár, Rational curves on algebraic varieties. Springer 1996. MR 98c:14001 Zbl 0877.14012

[13] A. Lanteri, H. Maeda, Ample vector bundles with sections vanishing on projective spaces or quadrics. Internat. J. Math. 6 (1995), 587-600. MR 96d:14039 Zbl 0876.14027

[14] A. Lanteri, H. Maeda, Geometrically ruled surfaces as zero loci of ample vector bundles. Forum Math. 9 (1997), 1-15. MR 97i:14027 Zbl 0876.14026

[15] S. Mori, Threefolds whose canonical bundles are not numerically effective. Ann. of Math. (2) 116 (1982), 133-176. MR 84e:14032 Zbl 0557.14021

[16] E. Sato, Z. Yicai, Smooth 4-folds which contain a $\mathbf{P}^{1}$-bundle as an ample divisor. Manuscripta Math. 101 (2000), 313-323. MR 2001a:14043 Zbl 01441728

[17] A. J. Sommese, Submanifolds of Abelian varieties. Math. Ann. 233 (1978), 229-256. MR 57 \#6524 Zbl 0381.14007

[18] M. Szurek, J. A. Wiśniewski, Fano bundles over $\mathbb{P}^{3}$ and $\mathbb{Q}^{3}$. Pacific J. Math. 141 (1990), 197-208. MR 91g:14036 Zbl 0705.14016

[19] J. A. Wiśniewski, On contractions of extremal rays of Fano manifolds. J. Reine Angew. Math. 417 (1991), 141-157. MR 92d:14032 Zbl 0721.14023

Received February 13, 2001; revised May 21, 2001 and June 25, 2001

M. Andreatta, G. Occhetta, Dipartimento di Matematica, Università degli Studi di Trento, Via Sommarive 14, I-38100 Povo (TN), Italy

Email: \{andreatt,occhetta\}@science.unitn.it 